\title{
Robust proactive eavesdropping in UAV-enabled wireless communication networking
}

\author{
Wei Wu' ${ }^{1}$ Yujing Wang ${ }^{2}$, Jianlin $\mathrm{Mo}^{3 *}$ and Jian Liu ${ }^{4}$
}

\begin{abstract}
In this paper, we considered a robust beamforming design problem for a proactive eavesdropping via jamming, in which a full-duplex legitimate monitor tries to eavesdrop on a suspicious communication link between the secondary pairs in an unmanned aerial vehicle (UAV)-enabled cognitive radio network. Due to the channel estimation errors, we assume that the channel state information is not perfectly known. To ensure the successful eavesdropping, jamming signals are designed to disrupt the suspicious receivers and the primary receiver at the same time, which should have the good tradeoff between those two effects. We aimed to maximize the achievable eavesdropping rate under the transmitting power constraint at the legitimate monitor and the interference temperature constraint at the primary receiver, which was formulated as a non-convex problem with infinite constraints. We firstly transform the original problem into a simplified one with finite constraints. Then, an analytical solution in significant low-complexity is proposed by decomposing the simplified problem. Numerical results are finally presented to evaluate the performance of our proposed schemes in UAV-enabled wireless communication networking.
\end{abstract}

Keywords: Unmanned aerial vehicles, Robust, Cognitive radio, Proactive eavesdropping, Beamforming

\section{Introduction}

Unmanned aerial vehicles (UAVs) have attracted a large amount of attention over recent years[1-3]. Their applications, such as surveillance, aerospace imaging, and cargo transportation, are widely studied. Extensive researches are also devoted to the use of drones as different types of wireless communication platforms, such as aeronautical mobile base stations (BS) $[4,5]$, mobile relays [6], and flight cloud computing $[7,8]$.

In a UAV-assisted network, UAVs are deployed to provide wireless connectivity to ground users[9]. In practice, UAVs can move freely in three-dimensional space for better performance. Due to UAVs' high mobility, the deployment of drone-assisted communication systems is more flexible, which makes them particularly suitable for on-demand applications or accidents. Furthermore, the UAV-ground link is more likely to have a line-of-sight

\footnotetext{
*Correspondence: mjl@abtc.edu.cn

${ }^{3}$ Electronic Information and Automation College of ABA, 623002 Sichuan, China

Full list of author information is available at the end of the article
}

(LoS) channel than the ground-to-ground link in the terrestrial system; thus, it can provide a higher link capacity. In addition, UAV-assisted communication provides additional design freedom for performance improvements by dynamically optimizing the UAV position to best meet communication requirements [10].

However, an inevitable problem in UAV-assisted network is the scarcity of wireless spectrum. Cognitive radio (CR), a promising technology to overcome spectrum overcrowding and scarcity, is considered a key technology to meet the critical requirements. UAVs may need to share the spectrum with existing wireless devices (e.g., cellular mobiles on the ground) for communications[11-13].

Wireless security has attracted a lot of researchers' attention recently, while the extensive work were often proposed to secure wireless communications against eavesdropping as illegitimate attacks [14-16]. In the UAV-assisted network, UAV wireless connection is an easy point of target when there is no strong protection against malicious hackers. Some effective means, such as encrypted channel and redundant coding, are widely used to enhance the security while safeguarding 
UAV from unauthorized access and disruption. However, criminals or terrorists may potentially use UAV-enabled wireless communications to establish suspicious links for crimes and terrorism $[17,18]$. Therefore, more and more demands were proposed for legitimate organizations, such as government agencies, e.g., to eavesdrop these suspicious links $[19,20]$. We thus intend to address the issue about the proactive eavesdropping in UAV-enabled wireless communication networking. It is worth noting that the flight trajectory and the placement of the UAV can be optimized for improving the communication performance, respectively. However, the mobility of UAVs is controlled by a suspicious user instead of the legitimate eavesdropper. In such a scenario, the proactive eavesdropper is a independent user, and the CSI between the eavesdropper and the suspicious transmitter can be estimated in a near-instantaneous manner[21, 22]. By contrast, the CSI between the eavesdropper and the receivers (primary receiver and secondary receiver) cannot be extracted. Therefore, we can assume that we know the statistical information of the channel between the eavesdropper and the receivers[23, 24].

The average eavesdropping rate over Rayleigh fading channels, defined as the suspicious communication rate multiplied by the non-outage probability, is maximized in [25]. Furthermore, it is considered to maximize the eavesdropping non-outage probability and the relative eavesdropping rate by optimizing the jamming power allocation over different fading states in [26]. On the other hand, multi-antenna wireless legitimate surveillance systems with non-ignored loop-channel are presented in [27]. It is worth noting that the aforementioned works on wireless surveillance mainly focus on the three-node systems with the suspicious parts and the legitimate monitor. The proactive eavesdropping in the scenario of CR network has not been investigated hitherto. In this paper, the suspicious users are set as the secondary users in a CR network; meanwhile, a full-duplex legitimate monitor tries to decode the messages in the suspicious link. The main contributions are summarized as follows:

1. We propose an efficient jamming scheme for proactive eavesdropping in the new UAV-enabled suspicious communication scenario with partial CSI between ST and SR, ST and PR, E and SR, and ST and E, which is shown in Fig. 1. The total interference power to PR caused by E and ST should be controlled below a threshold for all the possible channel realization within an uncertainty set.

2. We formulate an optimization problem to maximize the achievable eavesdropping rate by optimizing the jamming vector. First, the original problem with infinite constrains is transformed into a problem with finite constraints. Then, an analytical solution in closed-form or significant low-complexity is presented.

3. Numerical results reveal that the uncertainty of the channels influences the eavesdropping rate and the eavesdropping probability to a large extent. Furthermore, it is also certificated that our proposed solution has the better performance over the traditional solutions.

The rest of this paper is organized as follows. Section 2 explains the main methods of this paper. Section 3 introduces the system model of robust legitimate eavesdropping in a CR network, and then formulates the eavesdropping beamforming design problem. Section 4 divides the original problem into three sub-problems and

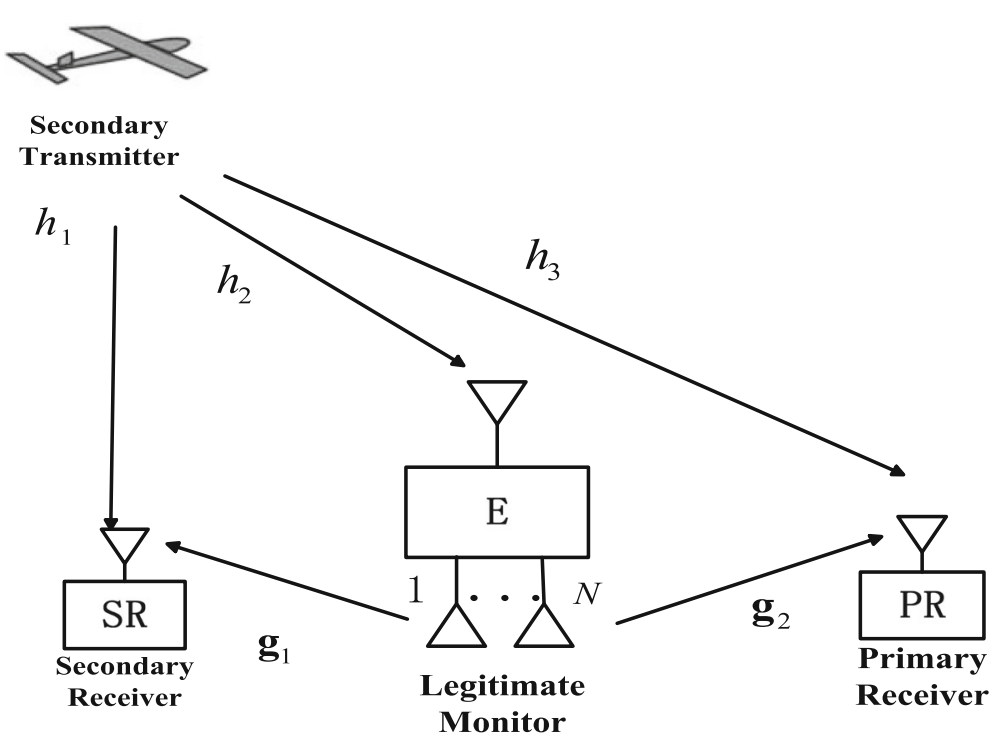

Fig. 1 System model 
proposes the optimal solution to the problems in a closedform. Section 5 provides the simulation results. Finally, Section 6 concludes the paper.

\section{Methods}

In a UAV-based wireless communication network, air-toground channel is an LoS channel that easily acquires channel knowledge. However, when a monitor attempts to monitor a terrestrial user, the channel between the monitor and the ground user is still affected by the complex environment on the ground. In this case, we assume that its statistical information is known and attempts to obtain a robust design. In such a scenario, we need to establish a robust optimization problem, which commits to get the best solution in the worst case.

The robust optimization problem involves infinite constraints. One common method to solve such problem is to use the S-procedure lemma to convert the infinite constraints. The optimal solution is obtained by decomposing the optimal matrix, which should permit that its rank equals to 1 . However, the drawback of this method is the high computational complexity. In this paper, we directly consider the worst case of the target and the boundary condition of the constrained space, then convert infinite constraints into finite constraints. Through the transformation of the triangle inequality, the semi-closed form solution of the original problem is finally obtained. The superiority of this approach lies in lower computational complexity and clear physical meaning.

\section{System model and problem formulation}

As shown in Fig. 1, we consider a suspicious wireless communication link from a secondary transmitter ST to a secondary receiver SR in a CR network, where a legitimate monitor $\mathrm{E}$ is intended to eavesdrop this link via jamming. ST and SR are both equipped with single antenna, and $E$ is deployed with one antenna for eavesdropping (receiving) and $N$ antennas for jamming (transmitting). When the primary user is inactive, it is clearly not the effect of the eavesdropping process. If the primary user is active, it is worth noting that the transmission information of the primary user in CR communication is useless for SR and E, so it can be treated as background noise. The scheme of the proactive eavesdropping would not be influenced by the primary user. The channels from ST to SR, from ST to $E$, from $S T$ to $P R$, from $E$ to $S R$, and from $E$ to $P R$ are denoted by $h_{1}, h_{2}, h_{3}, \mathbf{g}_{1}$, and $\mathbf{g}_{2}$, respectively. It is worth noting that downlink communication channels where the UAV sends messages to the ground users are dominated by line-of-sight (LoS) links, and the Doppler effect due to the UAV's mobility is assumed to be perfectly compensated. Therefore, the channel power gain from the UAV to each ground user follows the free-space path loss model given by

$$
h_{k}=\beta_{0} d_{k}^{-2}, k \in\{1,2,3\},
$$

where $\beta_{0}$ denotes the channel power gain at the reference distance $d_{0}=1$ meter $(\mathrm{m})$, and $d_{k}$ denotes the distance between ST and each ground user.

The legitimate monitor can operate in a full-duplex mode to enable simultaneous eavesdropping and jamming. Furthermore, we assume that the receiving antenna and the transmitting antennas at $\mathrm{E}$ are isolated [28], so that the self-interferences can be perfectly canceled at $\mathrm{E}$. E obtains the perfect CSI on the channel $h_{2}$ as a receiver. However, due to the channel estimation errors and the limited cooperation between ST, SR, and E, it is assumed that the legal monitor does not know the CSI on the channel $h_{1}, h_{3}, \mathbf{g}_{1}$, and $\mathbf{g}_{2}$ perfectly. Instead, it is reasonable to assume that $\mathrm{E}$ has the knowledge which is bounded within the uncertain sets described by the following regions:

$$
\eta_{e i}=\left\{\mathbf{g}_{i} \mid \mathbf{g}_{i}=\hat{\mathbf{g}}_{i}+\Delta \mathbf{g}_{i}, \Delta \mathbf{g}_{i}^{H} \Delta \mathbf{g}_{i} \leq \varepsilon_{e i}\right\}, i \in\{1,2\}
$$

where $\hat{\mathbf{g}}_{1}$ and $\hat{\mathbf{g}}_{2}$ are the estimators of the channels at E; $\Delta \mathbf{g}_{1}, \Delta \mathbf{g}_{2}$ are the corresponding channel estimation errors. $\varepsilon_{e 1}, \varepsilon_{e 2}$ determine the quality of the CSI estimations.

We consider that ST transmits with the power $P_{S}$, which satisfies $P_{S} \leq P_{t}$. Moreover, for any $\mathbf{w}$ satisfying $\|\mathbf{w}\|^{2} \leq P_{e}$ as the beamforming vector at $E$, the interference to $P R$ due to the suspicious transmission and the jamming signals sent by E can be expressed as: IT $=P_{S}\left\|h_{3}\right\|^{2}+\left\|\mathbf{g}_{2}^{H} \mathbf{w}\right\|^{2}$.

To ensure reliable detection at SR, adaptive rate transmission is adopted at ST based on the channel perceived at SR. For any fixed $\mathbf{w}$, the robust transmission rate at ST is formulated as:

$$
R_{S}(\mathbf{w})=\min _{\mathbf{g}_{1} \in \eta_{e 1}} \log _{2}\left(1+\frac{P_{S}\left\|h_{1}\right\|^{2}}{\left\|\mathbf{g}_{1}^{H} \mathbf{w}\right\|^{2}+\sigma^{2}}\right),
$$

where $\sigma^{2}$ refers to the variance of the Gaussian noises. In this case, the transmitting power at $\mathrm{ST}$ is given by the following Lemma.

Lemma 1 For any fixed $\mathbf{w}$, the robust transmitting power at ST is:

$$
P_{S}(\mathbf{w})=\min \left(\min _{\mathbf{g}_{2} \in \eta_{e 2}} \frac{\left(\Gamma-\left\|\mathbf{g}_{2}^{H} \mathbf{w}\right\|^{2}\right)^{+}}{\left\|h_{3}\right\|^{2}}, P\right),
$$

where $(x)^{+} \triangleq$ tolerable average interference at PR [29], which is called the interference temperature limit. 
Proof For any fixed $\mathbf{w}$, it can be observed that the channel capacity of the suspicious link is limited by two constraints: one is the transmit power constraint at ST; the other is the interference temperature constraint at SR. The following problem is considered to obtain the maximum robust transmission rate of the suspicious link:

$$
\begin{aligned}
\max _{P_{S}} & \min _{\mathbf{g}_{1} \in \eta_{e 1}} \frac{P_{S}\left\|h_{1}\right\|^{2}}{\left\|\mathbf{g}_{1}^{H} \mathbf{w}\right\|^{2}+\sigma^{2}} \\
\text { s.t. } & P_{S}\left\|h_{3}\right\|^{2}+\left\|\mathbf{g}_{2}^{H} \mathbf{w}\right\|^{2} \leq \Gamma, \forall \mathbf{g}_{2} \in \eta_{e 2}, h_{3} \in \eta_{a 3}, \\
& 0 \leq P_{S} \leq P_{t} .
\end{aligned}
$$

For problem (5), at least one constraint is active since the objective function increases with the increase of $P_{S}$. The optimal solution to (5) can be expressed as (4). The proof is thus completed.

On the other hand, the capacity of the eavesdropping channel from ST to E is:

$$
R_{E}(\mathbf{w})=\log _{2}\left(1+\frac{P_{S}(\mathbf{w})\left\|h_{2}\right\|^{2}}{\sigma^{2}}\right) .
$$

According to [30], the legitimate monitor can successfully eavesdrop the suspicious communication if and only if $R_{E}(\mathbf{w}) \geq R_{S}(\mathbf{w})$, since in this case the legitimate monitor can decode the information sent by ST with arbitrary small error probability. As a result, the effective eavesdropping rate is given by $R_{e v}(\mathbf{w})=R_{S}(\mathbf{w})$. On the other hand, if $R_{E}(\mathbf{w})<R_{S}(\mathbf{w})$, it is impossible for $\mathrm{E}$ to decode the information from ST without any error. In this case, the eavesdropping rate is defined as $R_{e v}(\mathbf{w})=0$. In summary, the effective eavesdropping rate can be expressed as:

$$
R_{e v}(\mathbf{w})=\left\{\begin{array}{ll}
R_{S}(\mathbf{w}) & \text { if } R_{E}(\mathbf{w}) \geq R_{S}(\mathbf{w}) \\
0 & \text { otherwise }
\end{array} .\right.
$$

The objective of the legitimate monitor is to maximize the eavesdropping rate $R_{e v}(\mathbf{w})$ by optimizing the beamforming vector $\mathbf{w}$. This problem is thus formulated as the following:

$$
\begin{aligned}
& \max _{\mathbf{w}} R_{e v}(\mathbf{w}) \\
& \text { s.t. } P_{S}(\mathbf{w})\left\|h_{3}\right\|^{2}+\left\|\mathbf{g}_{2}^{H} \mathbf{w}\right\|^{2} \leq \Gamma, \forall \mathbf{g}_{2} \in \eta_{e 2}, \\
& \quad\|\mathbf{w}\|^{2} \leq P_{e} .
\end{aligned}
$$

\section{Optimal solution}

First, we propose that $\mathbf{w}=\sqrt{p} \mathbf{v}$, where $p$ is the optimal transmit power satisfying $p \leq P_{e}$ and $\|\mathbf{v}\|^{2}=1$. Then, based on the definition in (7), the objective function can be replaced by $R_{S}(\mathbf{v}, p)$ with an additional constraint $R_{E}(\mathbf{v}, p) \geq R_{S}(\mathbf{v}, p)$. Meanwhile, by substituting (3) and (6) into (8), the original problem (8) can be expressed as

$$
\begin{aligned}
& \max _{p, \mathbf{v}} \min _{\mathbf{g}_{1} \in \eta_{e 1}, h_{1} \in \eta_{a 1}} \frac{P_{S}(\mathbf{v}, p)\left\|h_{1}\right\|^{2}}{p\left\|\mathbf{g}_{1} \mathbf{v}\right\|^{2}+\sigma^{2}} \\
& \text { s.t. } P_{S}(\mathbf{v}, p)\left\|h_{3}\right\|^{2}+p\left\|\mathbf{g}_{2} \mathbf{v}\right\|^{2} \leq \Gamma, \forall \mathbf{g}_{2} \in \eta_{e 2}, \\
& \quad \frac{\left\|h_{2}\right\|^{2}}{\sigma^{2}} \geq \frac{\left\|h_{1}\right\|^{2}}{p\left\|\mathbf{g}_{1} \mathbf{v}\right\|^{2}+\sigma^{2}}, \forall \mathbf{g}_{1} \in \eta_{e 1}, \\
& p \leq P_{e} .
\end{aligned}
$$

Note that the problem (9) is equivalent to the problem (8) due to the logarithmic transformation between rate and signal-to-interference-plus-noise ratio (SINR).

Remark 1 An important observation is that the constraint (9a) in the above problem remains invariant when $\hat{\mathbf{g}}_{2}$ undergoes any arbitrary rotation. Without loss of generality, we assume that $\hat{\mathbf{g}}_{2}$ and $\hat{\mathbf{g}}_{1}$ have the same phase, i.e., $\operatorname{Im}\left\{\hat{\mathbf{g}}_{2}^{H} \hat{\mathbf{g}}_{1}\right\}=0$, then we define the angle between $\hat{\mathbf{g}}_{2}$ and $\hat{\mathbf{g}}_{1}$ as $\alpha: \alpha=\arccos \left(\frac{\hat{\mathbf{g}}_{2}^{H} \hat{\mathbf{g}}_{1}}{\left\|\mathbf{g}_{2}^{H}\right\|\left\|\hat{\mathbf{g}}_{1}\right\|}\right)$.

Since problem (9) is subject to an infinite number of constraints. The traditional method about $\mathrm{S}$ procedure [31] could be applied to transform the problem (9) into a semidefinite program. However, in this paper, we are going to transform the original problem into a problem with finite constraints to get analytical results. In the following section, we firstly study several important constraints about problem (9). Then, we will analyze for them.

By assuming that $\mathbf{g}_{i 1}=\arg \max \mathbf{g}_{i}^{H} \mathbf{v} \mathbf{v}^{H} \mathbf{g}_{i}, i \in\{1,2\}$, where $\mathbf{g}_{i} \in \eta_{e i}$. We have $\mathbf{g}_{i 1}=\hat{\mathbf{g}}_{i}+\sqrt{\varepsilon_{e i}} \rho \mathbf{v}, \rho=$ $\frac{\hat{\mathbf{g}}_{i}^{H} \mathbf{v}}{\left\|\hat{\mathbf{g}}_{i}^{H} \mathbf{v}\right\|}$. The proof can be obtained by the simplified version of Lemma 2 in [32]. Moreover, we assume $\mathbf{g}_{i 0}=$ $\arg \min \mathbf{g}_{i}^{H} \mathbf{v} \mathbf{v}^{H} \mathbf{g}_{i}, \mathbf{g}_{i 0}=\hat{\mathbf{g}}_{i}-\sqrt{\varepsilon_{e i}} \rho \mathbf{v}, \rho=\frac{\hat{\mathbf{g}}_{i}^{H} \mathbf{v}}{\left\|\hat{\mathbf{g}}_{i}^{H} \mathbf{v}\right\|}$ is proposed.

Lemma 2 Problem (9) has the same optimal solution with the following problem:

$$
\begin{aligned}
& \max _{\mathbf{v}, p} \frac{P_{S}(\mathbf{v}, p)\left\|h_{1}\right\|^{2}}{p\left\|\mathbf{g}_{11}^{H} \mathbf{v}\right\|^{2}+\sigma^{2}} \\
& \text { s.t. } P_{S}(\mathbf{v}, p)\left\|h_{3}\right\|^{2}+p\left\|\mathbf{g}_{2}^{H} \mathbf{v}\right\|^{2} \leq \Gamma, \\
& \quad \frac{\left\|h_{2}\right\|^{2}}{\sigma^{2}} \geq \frac{\left\|h_{1}\right\|^{2}}{p\left\|\mathbf{g}_{10}^{H} \mathbf{v}\right\|^{2}+\sigma^{2}}, \\
& p \leq P_{e} .
\end{aligned}
$$

Proof First, we denote the objective function of problem (9) and (10) as $f_{1}$ and $f_{2}$, respectively. It can be observed that $\mathbf{g}_{11} \in \eta_{e 1}$. So, it can be satisfied that $f_{1} \leq f_{2}$. On 
the other hand, it can be proved by contradiction that $f_{2} \leq f_{1}$. We assume that there exist another parameters $\mathbf{g}_{12} \in \eta_{e 1}$ satisfying $f_{2}>\frac{P_{S}\left\|h_{1}\right\|^{2}}{p\left\|\mathbf{g}_{12}^{H} \mathbf{v}\right\|^{2}+\sigma^{2}}$. It can be obtained that $\left\|\mathbf{g}_{12}^{H} \mathbf{v}\right\|^{2} \leq\left\|\mathbf{g}_{11}^{H} \mathbf{v}\right\|^{2}$, which contradicts our presumption, i.e., $f_{1}=f_{2}$. Similarly, for problem (9), only the constraints in problem (10) are active constraints. The proof is then completed.

Moreover, the transmit power at ST can also be transformed into

$$
P_{S}(\mathbf{v}, p)=\min \left(\frac{\left(\Gamma-p\left\|\mathbf{g}_{21}^{H} \mathbf{v}\right\|^{2}\right)^{+}}{\left\|h_{3}\right\|^{2}}, P_{t}\right) .
$$

Then, we will analyze for the constraints of problem (10). For (10c), the following lemma is proposed:

Lemma 3 If the optimal solution to problem (9) satisfies that $p \neq 0$, the constraint (10c) could rewrite as

$$
\frac{\left\|h_{2}\right\|^{2}}{\sigma^{2}}=\frac{\left\|h_{1}\right\|^{2}}{p\left\|\mathbf{g}_{10}^{H} \mathbf{v}\right\|^{2}+\sigma^{2}}
$$

Proof Suppose $\left\{\mathbf{v}_{0}, p_{0}\right\}$ is the optimal solution of problem (10) subject to (10a) and (10b) with $p_{0} \neq 0$ satisfying $\left\|h_{2}\right\|^{2} / \sigma^{2}>\left\|h_{1}\right\|^{2} /\left(p_{0}\left\|\mathbf{g}_{1}^{H} \mathbf{v}_{0}\right\|^{2}+\sigma^{2}\right)$. There is another $p_{1}=c p_{0}(0<c<1$ is a scalar $)$ satisfying $\left\|h_{2}\right\|^{2} / \sigma^{2}=\left\|h_{1}\right\|^{2} /\left(p_{1}\left\|\mathbf{g}_{1}^{H} \mathbf{v}_{0}\right\|^{2}+\sigma^{2}\right)$. Then, it is easy to obtain that $\left\{\mathbf{v}_{0}, p_{1}\right\}$ also satisfies (10a) and (10b). Therefore, from (10), $\left\{\mathbf{v}_{0}, p_{1}\right\}$ is a better solution since $p_{1} \leq p_{0}$ and $P_{S}(\mathbf{v}, p)$ is an non-decreasing function with respect to $p$. This result contradicts our presumption.

For (10a), two cases are discussed corresponding to the conditions that if (10a) is a equality or not. When the optimal $\left\{\mathbf{v}^{*}, p^{*}\right\}$ causes that (10a) is not a equality, $P_{S}(\mathbf{v}, p)=$ $P_{t}$ is obtained due to the fact that another picking of $P_{S}(\mathbf{v}, p)$ only supports for the equality of (10a). Then, sub-problem 1 (SP1) is proposed

$$
\begin{aligned}
& (\mathrm{SP} 1): \max _{\mathbf{v}, p} \frac{P_{t}\left\|h_{1}\right\|^{2}}{p\left\|\mathbf{g}_{11}^{H} \mathbf{v}\right\|^{2}+\sigma^{2}} \\
& \text { s.t. } \frac{\left\|h_{2}\right\|^{2}}{\sigma^{2}}=\frac{\left\|h_{1}\right\|^{2}}{p\left\|\mathbf{g}_{10}^{H} \mathbf{v}\right\|^{2}+\sigma^{2}}, \\
& p \leq P_{e} .
\end{aligned}
$$

If $\left\{\mathbf{v}_{1}, p_{1}\right\}$, which is the optimal solution of problem (13), satisfies $P_{t}\left\|h_{3}\right\|^{2}+p_{1}\left\|\mathbf{g}_{21}^{H} \mathbf{v}_{1}\right\|^{2}<\Gamma,\left\{\mathbf{v}_{1}, p_{1}\right\}$ is the optimal solution for problem (9). Otherwise, constraint (10a) is a equality for the optimal solution of (9).
In the case when constraint (10a) is a equality, another two SPs are proposed

$$
\begin{gathered}
\text { (SP2) : } \max _{\mathbf{v}, p} \frac{\left(\Gamma-p\left\|\mathbf{g}_{21}^{H} \mathbf{v}\right\|^{2}\right)\left\|h_{1}\right\|^{2}}{\left(p\left\|\mathbf{g}_{11}^{H} \mathbf{v}\right\|^{2}+\sigma^{2}\right)\left\|h_{3}\right\|^{2}} \\
\text { s.t. } \frac{\left\|h_{2}\right\|^{2}}{\sigma^{2}}=\frac{\left\|h_{1}\right\|^{2}}{p\left\|\mathbf{g}_{10}^{H} \mathbf{v}\right\|^{2}+\sigma^{2}}, \\
p \leq P_{e} . \\
\text { (SP3) : } \max _{\mathbf{v}, p} \frac{P_{t}\left\|h_{1}\right\|^{2}}{p\left\|\mathbf{g}_{11}^{H} \mathbf{v}\right\|^{2}+\sigma^{2}} \\
\text { s.t. } P_{t}\left\|h_{3}\right\|^{2}+p\left\|\mathbf{g}_{21}^{H} \mathbf{v}\right\|^{2}=\Gamma, \\
\frac{\left\|h_{2}\right\|^{2}}{\sigma^{2}}=\frac{\left\|h_{1}\right\|^{2}}{p\left\|\mathbf{g}_{10}^{H} \mathbf{v}\right\|^{2}+\sigma^{2}}, \\
p \leq P_{e} .
\end{gathered}
$$

If the optimal solution of SP2, defined as $\left\{\mathbf{v}_{2}, p_{2}\right\}$, satisfies $0 \leq \Gamma-p_{2}\left\|\mathbf{g}_{21}^{H} \mathbf{v}_{2}\right\|^{2} \leq P_{t},\left\{\mathbf{v}_{2}, p_{2}\right\}$ is the optimal solution for problem (9). Else if $\Gamma-p_{2}\left\|\mathbf{g}_{21}^{H} \mathbf{v}_{2}\right\|^{2}<0$, problem (9) is not feasible. Otherwise, i.e., $\Gamma-p_{2}\left\|\mathbf{g}_{21}^{H} \mathbf{v}_{2}\right\|^{2}>$ $P_{t}$, the optimal solution of SP3, defined as $\left\{\mathbf{v}_{3}, p_{3}\right\}$, is the optimal solution for problem (9).

\subsection{Optimal solution for SP1}

Before we proceed to solve SP1, an important property is proposed as follows.

Remark 2 For $i \in\{1,2\}$, by applying the triangle inequality, the constraint can be transformed as follows

$$
\left\|\mathbf{g}_{i 0}^{H} \mathbf{v}\right\| \leq\left\|\mathbf{g}_{i}^{H} \mathbf{v}\right\| \leq\left\|\mathbf{g}_{i 1}^{H} \mathbf{v}\right\| .
$$

And

$$
\begin{aligned}
& \left\|\mathbf{g}_{i 0}^{H} \mathbf{v}\right\|=\left\|\left(\hat{\mathbf{g}}_{i}^{H}-\sqrt{\varepsilon_{e i}} \rho \mathbf{v}^{H}\right) \mathbf{v}\right\| \geq\left\|\hat{\mathbf{g}}_{i}^{H} \mathbf{v}\right\|-\sqrt{\varepsilon_{e i}} \rho, \\
& \left\|\mathbf{g}_{i 1}^{H} \mathbf{v}\right\|=\left\|\left(\hat{\mathbf{g}}_{i}^{H}+\sqrt{\varepsilon_{e i}} \rho \mathbf{v}^{H}\right) \mathbf{v}\right\| \leq\left\|\hat{\mathbf{g}}_{i}^{H} \mathbf{v}\right\|+\sqrt{\varepsilon_{e i}} \rho .
\end{aligned}
$$

Then, for the optimal $\mathbf{v}^{*}$, it can be obtained that $\hat{\mathbf{g}}_{i}, \mathbf{v}^{*}$ have the same phase, i.e., $\operatorname{Re}\left\{\hat{\mathbf{g}}_{i}^{H} \mathbf{v}^{*}\right\} \geq 0, \operatorname{Im}\left\{\hat{\mathbf{g}}_{i}^{H} \mathbf{v}^{*}\right\}=0$. And it can be observed that $\rho=1,\left\|\mathbf{g}_{i 0}^{\bar{H}} \mathbf{v}^{*}\right\|=\hat{\mathbf{g}}_{i}^{H} \mathbf{v}^{*}-\sqrt{\varepsilon_{e i}}$, $\left\|\mathbf{g}_{i 1}^{H} \mathbf{v}^{*}\right\|=\hat{\mathbf{g}}_{i}^{H} \mathbf{v}^{*}+\sqrt{\varepsilon_{e i}}$.

By using Remark 2, SP1 can be transformed into

$$
\begin{aligned}
& \max _{p, \mathbf{v}} \frac{P_{t}\left(\left\|h_{1}\right\|\right)^{2}}{p\left(\hat{\mathbf{g}}_{1}^{H} \mathbf{v}+\sqrt{\varepsilon_{e 1}}\right)^{2}+\sigma^{2}} \\
& \text { s.t.p } \leq P_{e}, \\
& \quad \frac{\left\|h_{2}\right\|^{2}}{\sigma^{2}}=\frac{\left(\left\|h_{1}\right\|\right)^{2}}{p\left(\hat{\mathbf{g}}_{1}^{H} \mathbf{v}-\sqrt{\varepsilon_{e 1}}\right)^{2}+\sigma^{2}} .
\end{aligned}
$$

Substitute (19b) into the objective function of (19) and the optimal solution to problem (19) can be obtained if the following function 


$$
\begin{aligned}
f_{1}(\mathbf{v}) & =\frac{\delta\left(\hat{\mathbf{g}}_{1}^{H} \mathbf{v}+\sqrt{\varepsilon_{e 1}}\right)^{2}}{\left(\hat{\mathbf{g}}_{1}^{H} \mathbf{v}-\sqrt{\varepsilon_{e 1}}\right)^{2}} \\
& =\delta\left(1+\frac{2 \sqrt{\varepsilon_{e 1}}}{\hat{\mathbf{g}}_{1}^{H} \mathbf{v}-\sqrt{\varepsilon_{e 1}}}\right)^{2}
\end{aligned}
$$

is minimized, where $\delta=\frac{\left(\left\|h_{1}\right\|\right)^{2} \sigma^{2}}{\left\|h_{2}\right\|^{2}}-\sigma^{2}$ and $\delta>0$.

It is easy to obtain that the optimal solution to SP1 is

$$
\mathbf{v}_{1}=\frac{\hat{\mathbf{g}}_{1}}{\left\|\hat{\mathbf{g}}_{1}\right\|}, p_{1}=\frac{\delta}{\left(\hat{\mathbf{g}}_{1}^{H} \mathbf{v}_{1}-\sqrt{\varepsilon_{e 1}}\right)^{2}}
$$

if and only if $p_{1} \leq P_{e}$ is satisfied.

\subsection{Optimal solution for $\mathrm{sP2}$}

By using Remark 2, SP2 can be transformed into

$$
\begin{aligned}
& \max _{p, \mathbf{v}} \frac{\left(\Gamma-p\left(\hat{\mathbf{g}}_{2}^{H} \mathbf{v}+\sqrt{\varepsilon_{e 2}}\right)^{2}\right)\left(\left\|h_{1}\right\|\right)^{2}}{\left(p\left(\hat{\mathbf{g}}_{1}^{H} \mathbf{v}+\sqrt{\varepsilon_{e 1}}\right)^{2}+\sigma^{2}\right)\left(\left\|h_{3}\right\|\right)^{2}} \\
& \text { s.t. (19a),(19b). }
\end{aligned}
$$

Substitute (19b) into the objective function of (22) and it can be observed that the optimal solution to problem (22) can be obtained if the following function

$$
f_{2}(\mathbf{v})=\frac{\Gamma\left(\hat{\mathbf{g}}_{1}^{H} \mathbf{v}-\sqrt{\varepsilon_{e 1}}\right)^{2}-\delta\left(\hat{\mathbf{g}}_{2}^{H} \mathbf{v}+\sqrt{\varepsilon_{e 1}}\right)^{2}}{\delta\left(\hat{\mathbf{g}}_{1}^{H} \mathbf{v}+\sqrt{\varepsilon_{e b}}\right)^{2}+\sigma^{2}\left(\hat{\mathbf{g}}_{1}^{H} \mathbf{v}-\sqrt{\varepsilon_{e 1}}\right)^{2}} .
$$

is maximized.

In the following lemma, the optimal beamforming vector $\mathbf{v}$ at (23) is proposed with an important property.
Lemma 4 The optimal vector $\mathbf{v}$ for (23) is of the form $\alpha_{\nu} \hat{\mathbf{g}}_{\|}-\beta_{\nu} \hat{\mathbf{g}}_{\perp}, \alpha_{v}, \beta_{v} \in \mathbb{R}$, where $\hat{\mathbf{g}}_{\|}=\hat{\mathbf{g}}_{1} /\left\|\hat{\mathbf{g}}_{1}\right\|, \hat{\mathbf{g}}_{\perp}=$ $\mathbf{g}_{\perp} /\left\|\mathbf{g}_{\perp}\right\|, \mathbf{g}_{\perp}=\hat{\mathbf{g}}_{2}-\left(\hat{\mathbf{g}}_{\|}^{H} \hat{\mathbf{g}}_{2}\right) \hat{\mathbf{g}}_{\|}$.

Proof The proof of Lemma 4 could be divided into two parts. The first part is to prove that $\mathbf{v}$ is of the form $\alpha_{\nu} \hat{\mathbf{g}}_{\|}-$ $\beta_{v} \hat{\mathbf{g}}_{\perp}, \alpha_{v}, \beta_{v} \in \mathbb{C}$. The second part is to prove $\alpha_{v}, \beta_{v} \in \mathbb{R}$.

First, we assume that the vector $\mathbf{v}_{x}=\alpha_{\nu} \hat{\mathbf{g}}_{\|}-\beta_{\nu} \hat{\mathbf{g}_{0}}$ is the optimal vector at (23), where $\hat{\mathbf{g}_{\mathbf{0}}}{ }^{H} \hat{\mathbf{g}}_{\|}=0,\left\|\hat{\mathbf{g}_{\mathbf{0}}}\right\|=1$ and $\hat{\mathbf{g}_{0}} \neq \hat{\mathbf{g}}_{\perp}$. There is another vector $\mathbf{v}_{y}=\alpha_{\nu} \hat{\mathbf{g}}_{\|}-\beta_{\nu} \hat{\mathbf{g}}_{\perp}$. It can be observed that $f_{2}\left(\mathbf{v}_{x}\right) \leq f_{2}\left(\mathbf{v}_{y}\right)$ due to the fact that $\hat{\mathbf{g}}_{1}^{H} \mathbf{v}_{x}=\hat{\mathbf{g}}_{1}^{H} \mathbf{v}_{y}$ and $\hat{\mathbf{g}}_{2}^{H} \mathbf{v}_{x} \leq \hat{\mathbf{g}}_{2}^{H} \mathbf{v}_{y}$, where the equal sign is active if and only if $\beta_{1}=0$.

We next prove that $\alpha_{v}, \beta_{v} \in \mathbb{R}$. By using Remark 2, it can be obtained that $\mathbf{g}_{\perp}, \mathbf{g}_{\|}$and $\mathbf{v}$ have the same phase. i.e., $\alpha_{v}, \beta_{\nu} \in \mathbb{R}$ for $\alpha_{\nu} \hat{\mathbf{g}}_{\|}-\beta_{\nu} \hat{\mathbf{g}}_{\perp}$.

From Lemma 4, we learn that the optimal vector $\mathbf{v}$ is shown to lie in a two-dimensional (2-D) space spanned by $\hat{\mathbf{g}}_{\|}$and $\hat{\mathbf{g}}_{\perp}$. So, it can be searched on the space as depicted in Fig. 2.

It can be obtained from Fig. 2 that

$$
\hat{\mathbf{g}}_{1}^{H} \mathbf{v}=\left\|\hat{\mathbf{g}}_{1}\right\| \cos (\beta), \hat{\mathbf{g}}_{2}^{H} \mathbf{v}=\left\|\hat{\mathbf{g}}_{2}\right\| \cos (\alpha+\beta) .
$$

By substituting (24) into (23), we have

$$
f_{2}(\beta)=\frac{\Gamma\left(\left\|\hat{\mathbf{g}}_{1}\right\| \cos (\beta)-\sqrt{\varepsilon_{e 1}}\right)^{2}-\delta\left(\left\|\hat{\mathbf{g}}_{2}\right\| \cos (\alpha+\beta)+\sqrt{\varepsilon_{e 2}}\right)^{2}}{\delta\left(\left\|\hat{\mathbf{g}}_{1}\right\| \cos (\beta)+\sqrt{\varepsilon_{e 1}}\right)^{2}+\sigma^{2}\left(\left\|\hat{\mathbf{g}}_{1}\right\| \cos (\beta)-\sqrt{\varepsilon_{e 1}}\right)^{2}} .
$$

$f_{2}(\beta)$ is a single variable optimization problem. To find the optimal $\beta$, we should firstly observe that the feasible region for the variable $\beta$ is $[0, \pi / 2-\alpha]$. And $p=$

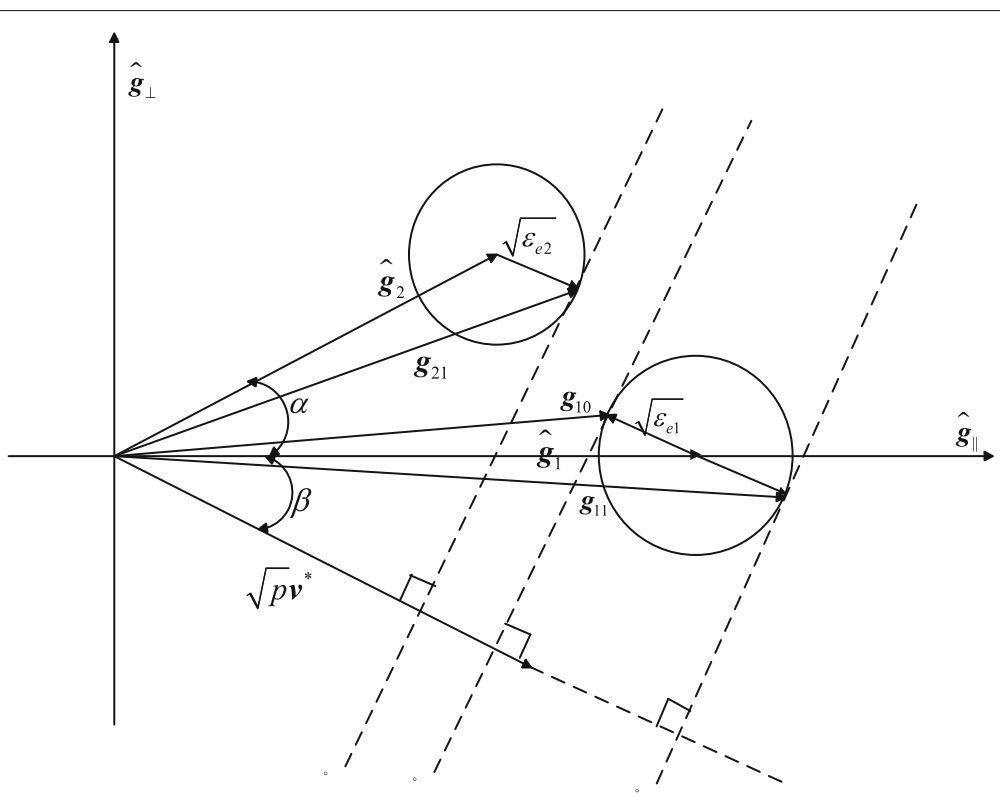

Fig. 2 The space spanned by $\hat{\boldsymbol{h}}_{\|}$and $\hat{\boldsymbol{h}}_{\perp}$ 
$\delta /\left(\left\|\hat{\mathbf{g}}_{1}\right\| \cos (\beta)-\sqrt{\varepsilon_{e 1}}\right)^{2} \leq P_{e}$ should be satisfied. So, $\beta$ is in the range of $\left[0, \min \left(\pi / 2-\alpha, \beta_{1}\right)\right]$, where $\beta_{1}$ satisfies $\left\|\hat{\mathbf{g}}_{1}\right\| \cos \left(\beta_{1}\right)=\max \left(\pi / 2-\alpha, \sqrt{\delta / P_{e}}+\sqrt{\varepsilon_{e 1}}\right)$. However, the first-order derivative of $f_{2}(\beta)$ is too complex to get a closed-form result. Then, the globally optimal solution of $f_{2}(\beta)$ could be searched for in the feasible region.

By using Lemma 4 , the optimal solution $\left\{\mathbf{v}_{2}, p_{2}\right\}$ can be obtained with $\beta_{2}$, which is the optimal solution of $f_{2}(\beta)$ :

$$
\begin{gathered}
\alpha_{v}=\cos \left(\beta_{2}\right), \beta_{v}=\sin \left(\beta_{2}\right), \\
p_{2}=\frac{\delta}{\left(\left\|\hat{\mathbf{g}}_{1}\right\| \cos \left(\beta_{2}\right)-\sqrt{\varepsilon_{e 1}}\right)^{2}} .
\end{gathered}
$$

\subsection{Optimal solution for SP3}

For SP3, Lemma 4 is also applicable, and the following equations are proposed:

$$
\begin{aligned}
p_{3}\left(\left\|\hat{\mathbf{g}}_{2}\right\| \cos (\alpha+\beta)+\sqrt{\varepsilon_{e 2}}\right)^{2} & =\delta_{1}, \\
p_{3}\left(\left\|\hat{\mathbf{g}}_{1}\right\| \cos (\beta)-\sqrt{\varepsilon_{e 1}}\right)^{2} & =\delta,
\end{aligned}
$$

where $\delta_{1}=\Gamma-P\left(\left\|\hat{h}_{3}\right\|+\sqrt{\varepsilon_{a 3}}\right)^{2}$.

It is easy to obtain $\left\{\beta_{3}, p_{3}\right\}$ by solving the equations at (26) with the regular mathematical tools and the optimal vector $\mathbf{v}_{3}$ is proposed as follows

$$
\mathbf{v}_{3}=\cos \left(\beta_{3}\right) \hat{\mathbf{g}}_{\|}-\sin \left(\beta_{3}\right) \hat{\mathbf{g}}_{\perp} .
$$

\section{Simulation results and discussions}

Simulation results are provided in this section, in which $\mathrm{E}$ is equipped with one antenna for receiving and $(N=4)$ antennas for transmitting. Meanwhile, ST, SR, and PR each use a single antenna. It is assumed the channels are drawn that each element is generated by independent CSCG variable distributed as $\mathcal{C N}(0,1)$ for $\hat{\mathbf{g}}_{1}$ and $\hat{\mathbf{g}}_{2}$, respectively. The corresponding distance is assumed to be $d_{1}=200 \mathrm{~m}, d_{2}=d_{3}=500 \mathrm{~m}$. For simplicity, the uncertainties of all channels are assumed to be $\varepsilon_{e 1}=\varepsilon_{e 2}=\varepsilon$, where $\varepsilon$ specifies the size of the uncertainty set.

Figure 3 illustrates robust eavesdropping rate versus the interference temperature limit at PR. $P_{t} / \sigma^{2}=P_{e} / \sigma^{2}=$ $10 \mathrm{~dB}$ while $\Gamma / \sigma^{2}$ is in the range of $[-10 \mathrm{~dB}, 10 \mathrm{~dB}]$. Note that $\varepsilon=0$ corresponds to the scenario when perfect knowledge of all the channels is known. When the constraint in Theorem 1 is not satisfied, $\left\{\mathbf{v}_{i}, p_{i}\right\}, i \in$ $\{2,3\}$ may be the optimal solution. In this case, robust eavesdropping rate will increase with the increase of $\Gamma$ until $\left\{\mathbf{v}_{1}, p_{1}\right\}$ is a feasible solution. Moreover, it can be observed that robust eavesdropping rate is higher when $\varepsilon$ is smaller, which can be explained that with more accurate CSI, E can better obtain the information about the suspicious link and achieve successful eavesdropping more effectively.

Figure 4 depicts the eavesdropping rate versus the maximum transmit power at $\mathrm{E}$. The parameter $P_{t} / \sigma^{2}$ is 10 $\mathrm{dB}$ while $\Gamma / \sigma^{2}$ is $0 \mathrm{~dB} . P_{e} / \sigma^{2}$ increases from $-5 \mathrm{~dB}$ to $10 \mathrm{~dB}$. Similarly, higher robust eavesdropping rate can be observed with smaller $\varepsilon$. The curves are increasing with the increase of $\Gamma$ until the upper bound is obtained.

Figure 5 illustrates the eavesdropping probability versus the interference temperature limit at PR, where the eavesdropping probability is denoted by the ratio of the

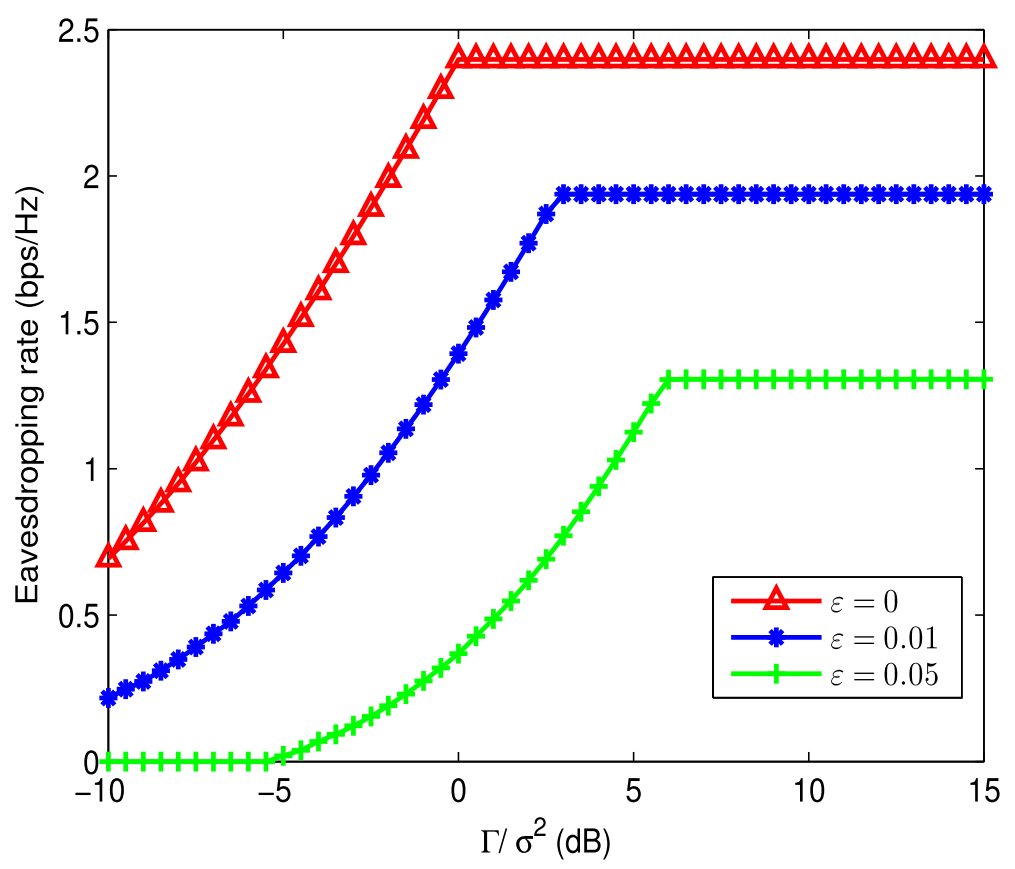

Fig. 3 Eavesdropping rate versus the interference temperature limit at PR 


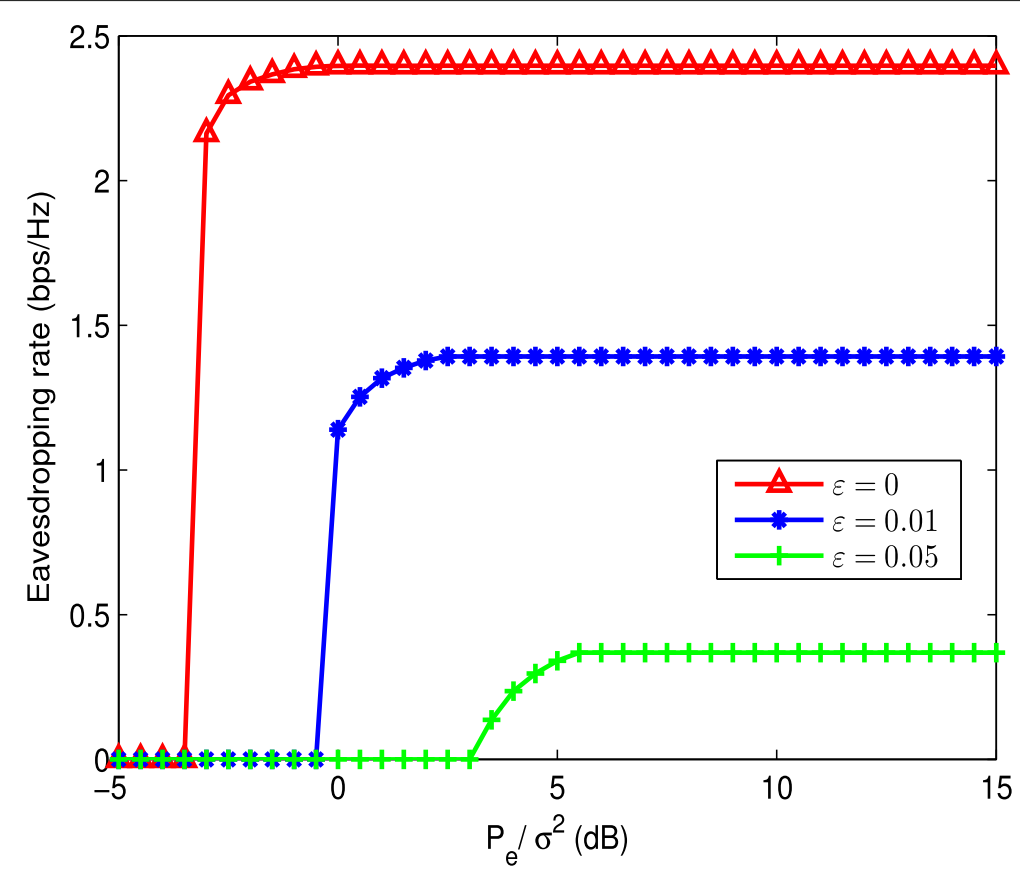

Fig. 4 Eavesdropping rate versus the maximum transmit power at $E$

successful eavesdropping to the sum eavesdropping, successful eavesdropping is defined as the cases that the eavesdropping rate can not be larger corresponding to the known conditions. $P_{t} / \sigma^{2}=P_{e} / \sigma^{2}=10 \mathrm{~dB}$ while $\Gamma / \sigma^{2}$ is in the range of $[-10 \mathrm{~dB}, 10 \mathrm{~dB}]$. Note that $\varepsilon=0$ corresponds to the scenario when perfect knowledge of all the channels is known. In such a scene, the eavesdropping probability gets the best performance. Moreover, it can be observed that robust eavesdropping probability increases to 1 more quickly when $\varepsilon$ is smaller, which can be explained that with more accurate CSI, E can better obtain the information about the suspicious link and achieve successful eavesdropping more effectively.

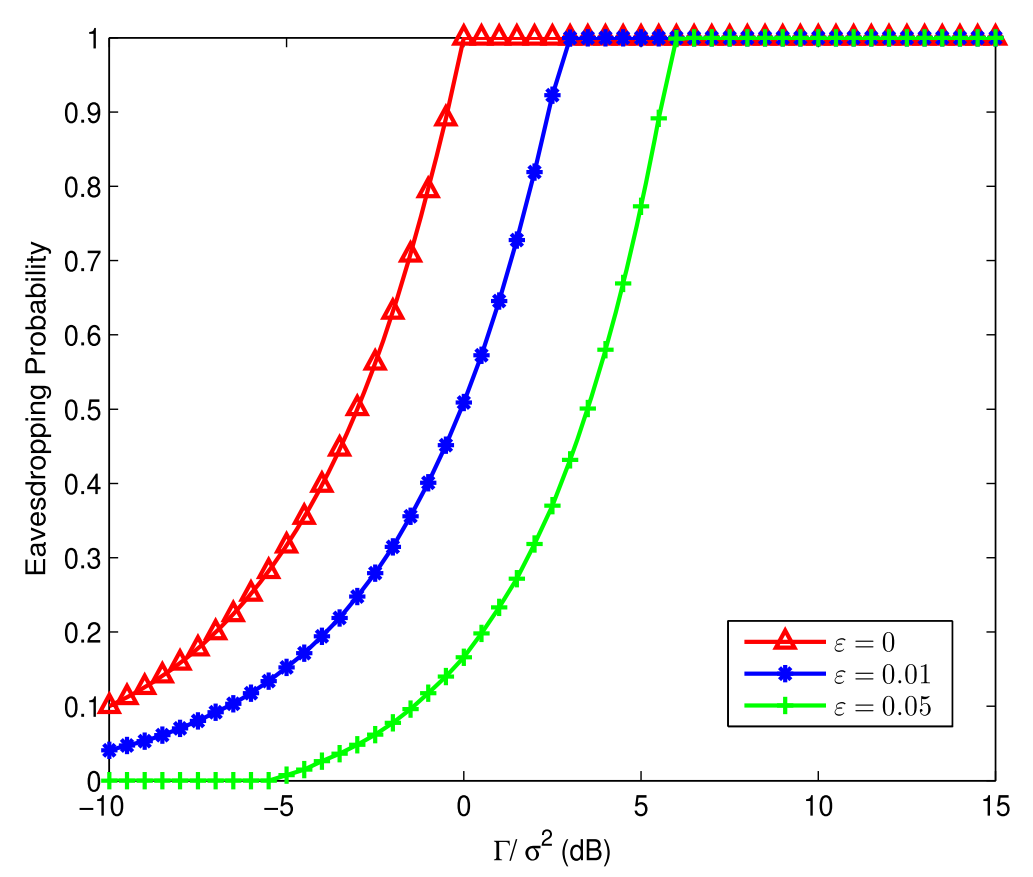

Fig. 5 Eavesdropping probability versus the interference temperature limit at PR 


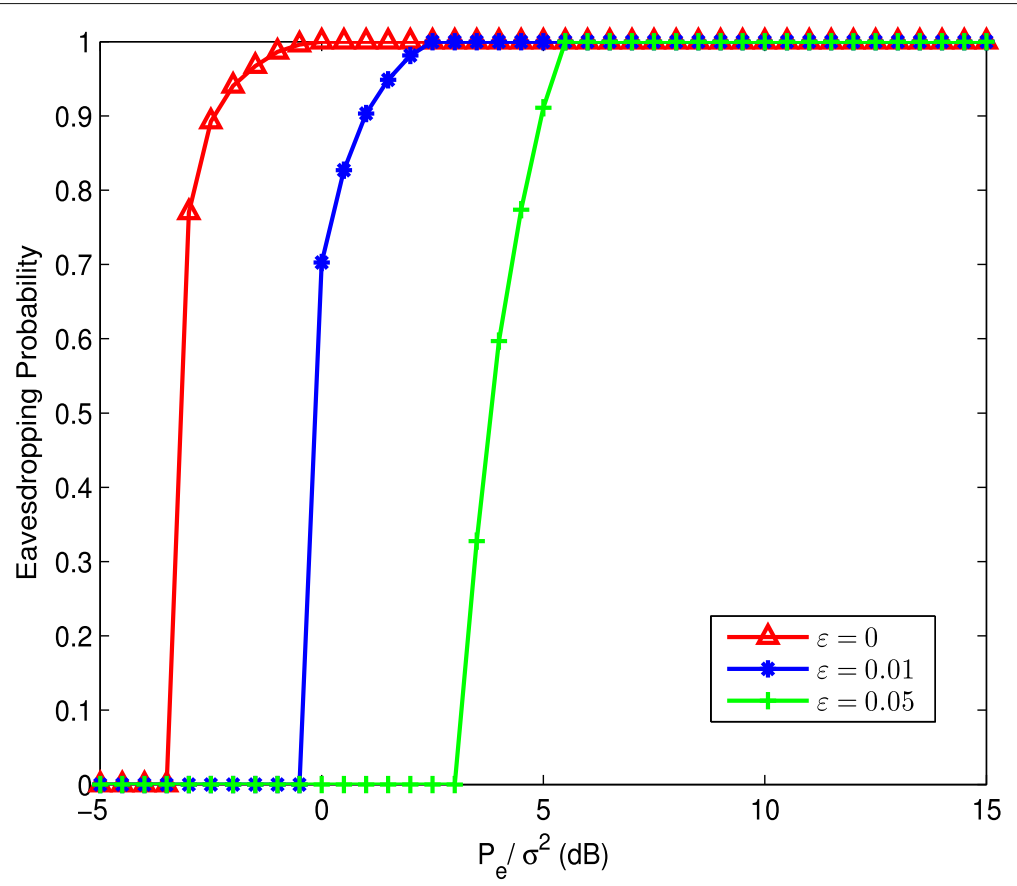

Fig. 6 Eavesdropping probability versus the maximum transmit power at $E$

Figure 6 depicts the eavesdropping probability versus the maximum transmit power at $\mathrm{E}$. The parameter $P_{t} / \sigma^{2}$ is $10 \mathrm{~dB}$ while $\Gamma / \sigma^{2}$ is $0 \mathrm{~dB} . P_{e} / \sigma^{2}$ increases from -5 $\mathrm{dB}$ to $10 \mathrm{~dB}$. Similarly, higher eavesdropping probability is obtained with smaller $\varepsilon$. The curves are increasing with the increase of $\Gamma$ until the upper bound is obtained.
Figure 7 depicts the eavesdropping rate versus the uncertainty of all channels. We compare our approach with the traditional solution in [33], which does not incorporate the robust cases. When $\varepsilon=0$, the CSI is completely known, and these two solutions have the same eavesdropping rate. With the increases of $\varepsilon$, our approach achieves better eavesdropping rate than the other one.

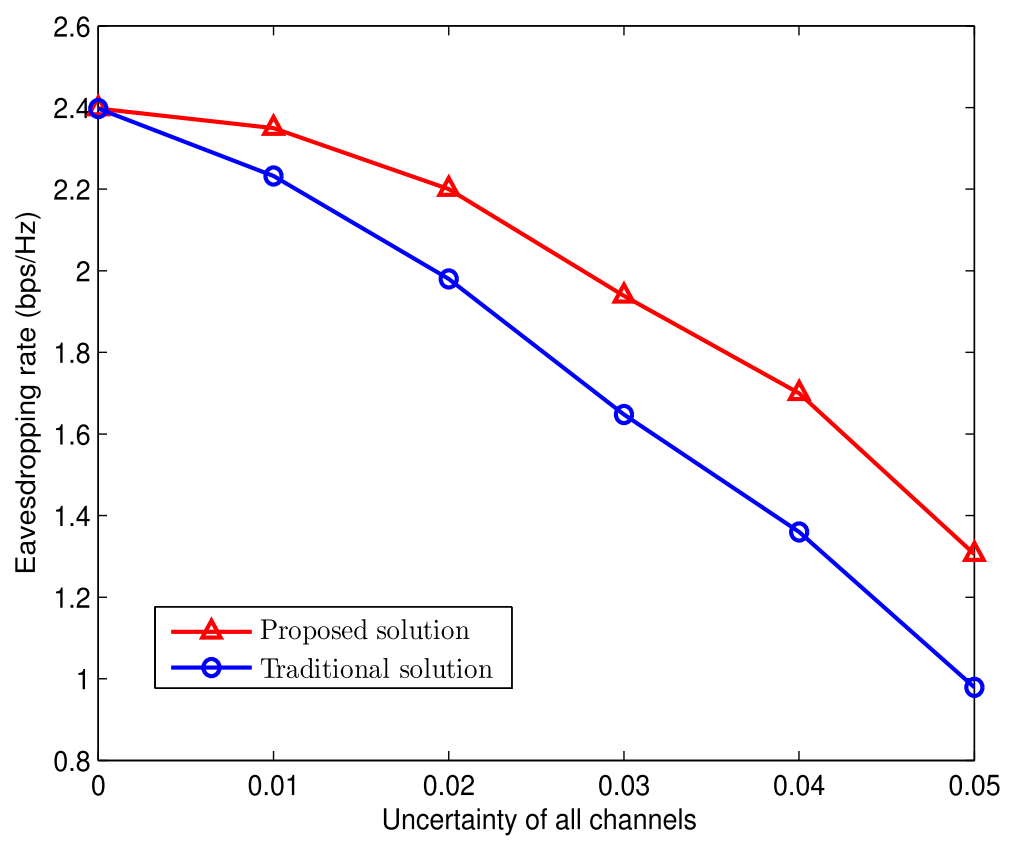

Fig. 7 Eavesdropping rate versus the uncertainty of all channels 
The gap also becomes larger due to the uncertainty of the CSI.

The parameter $P_{t} / \sigma^{2}$ is $10 \mathrm{~dB}$ while $\Gamma / \sigma^{2}$ is $0 \mathrm{~dB} . P_{e} / \sigma^{2}$ increases from $-5 \mathrm{~dB}$ to $10 \mathrm{~dB}$. Similarly, higher robust eavesdropping rate can be observed with smaller $\varepsilon$. The curves are increasing with the increase of $\Gamma$ until the upper bound is obtained.

\section{Conclusion}

This paper has investigated a new robust information surveillance problem under the UAV-enabled CR network. A proactive eavesdropping scheme has been considered to monitor the suspicious transmission between the suspicious UAV transmitter and the ground receiver via jamming. The full-duplex legitimate monitor adaptively optimizes the transmission beamforming vector to maximize the eavesdropping rate, which is formulated as a non-convex semi-infinite optimization problem. To solve it, we have transformed it into a problem with finite constraints, then the optimal solution in low-complexity were proposed. The numerical results show the superiority of our proposed schemes in UAV-enabled wireless communication networking.

\section{Abbreviations}

BS: Base station; CR: Cognitive radio; LoS: Line-of-sight; UAV: Unmanned aerial vehicle

\section{Acknowledgments}

Not applicable.

\section{Authors' contributions}

WW is the main author of the current paper. WW contributed to the development of the ideas, design of the study, theory, result analysis, and article writing. YW carried out the experimental work and the data collection and interpretation. JM finished the analysis and interpretation of data and drafted the manuscript. JL conceived and designed the experiments and undertook revision works of the paper. All authors read and approved the final manuscript.

\section{Funding}

This work was supported in part by the National Natural Science Foundation of China (61901231), the Natural Science Foundation of Jiangsu Province of China (BK20180757), the Project of Educational Commission of Jiangsu Province of China (18KJB510028), the Introducing Talent Research Start-Up Fund of Nanjing University of Posts and Telecommunications (NY218100, NY219072), and the Aba Prefecture Applied Technology Research and Development Fund Project (19YYJSYJ0035).

\section{Availability of data and materials}

Data sharing not applicable to this article as no data sets were generated or analyzed during the current study.

\section{Competing interests}

The authors declare that they have no competing interests.

\section{Author details}

${ }^{1}$ Nanjing University of Posts and Telecommunications, 210003 Nanjing, China. ${ }^{2}$ Dean's office of ABA, 623002 Sichuan, China. ${ }^{3}$ Electronic Information and Automation College of ABA, 623002 Sichuan, China. ${ }^{4}$ College of Information Engineering, Nanjing University of Finances and Economics, 210003 Nanjing, China.
Received: 25 September 2019 Accepted: 11 November 2019

Published online: 27 December 2019

\section{References}

1. C. Li, S. Zhang, P. Liu, F. Sun, J. M. Cioffi, L. Yang, Overhearing protocol design exploiting inter-cell interference in cooperative green networks. IEEE Trans. Veh. Technol. 65(1), 441-446 (2016)

2. R. Q. Hu, Y. Qian, An energy efficient and spectrum efficient wireless heterogeneous network framework for $5 \mathrm{G}$ systems. IEEE Commun. Mag. 52(5), 94-101 (2014)

3. W. Wu, F. Zhou, P. Li, P. Deng, B. Wang, V. C. M. Leung, in 2019 IEEE International Conference on Communications (ICC). Energy-efficient secure NOMA-enabled mobile edge computing networks, (Shanghai, 2019)

4. C. Li, P. Liu, C. Zou, F. Sun, J. M. Cioffi, L. Yang, Spectral-efficient cellular communications with coexistent one- and two-hop transmissions. IEEE Trans. Veh. Technol. 65(8), 6765-6772 (2016)

5. Y. Zeng, J. Lyu, R. Zhang, Cellular-connected UAV: potential, challenges, and promising technologies. IEEE Wirel. Commun. 26(1), 120-127 (2019)

6. C. Li, F. Sun, J. M. Cioffi, L. Yang, Energy efficient MIMO relay transmissions via joint power allocations. IEEE Trans. Circ. Syst. 61(7), 531-535 (2014)

7. W. Wu, X. Yin, P. Deng, T. Guo, B. Wang, Transceiver design for Downlink SWIPT NOMA Systems With Cooperative Full-Duplex Relaying. IEEE Access. 7, 33464-33472 (2019)

8. F. Zhou, Y. Wu, H. Sun, Z. Chu, in 2018 IEEE International Conference on Communications (ICC). UAV-enabled mobile edge computing: offloading optimization and trajectory design, (Kansas City, 2018). https://doi.org/10. 1109/icc.2018.8422277

9. C. Li, H. J. Yang, F. Sun, J. M. Cioffi, L. Yang, Adaptive overhearing in two-way multi-antenna relay channels. IEEE Sig. Process. Lett. 23(1), 117-120 (2016)

10. X. Hong, P. Liu, F. Zhou, S. Guo, Z. Chu, Resource allocation for secure UAV-assisted SWIPT systems. IEEE Access. 7, 8-24257 (2019)

11. W. Wu, B. Wang, Robust secrecy beamforming for wireless information and power transfer in multiuser MISO communication system. EURASIP J. Wirel. Commun. Netw. 1(2015), 161 (2015)

12. F. Zhou, Y. Wu, R. Q. Hu, Y. Qian, Computation rate maximization in UAV-enabled wireless powered mobile-edge computing systems. IEEE J. Sel. Areas Commun. 36(9), 1927-1941 (2018)

13. J. Cao, Z. Bu, Y. Wang, H. Yang, J. Jiang, H. Li, Detecting prosumer-community group in smart grids from the multiagent perspective. IEEE Trans. Syst. Man Cybern.-Syst. 49(8), 1652-1664 (2019)

14. Y. Liu, H.H. Chen, L. Wang, Physical layer security for next generation wireless networks: theories, technologies, and challenges. IEEE Commun. Surv. Tutor. 19(1), 347-375 (2016)

15. S. Y. Park, D. J. Love, Outage performance of multi-antenna multicasting for wireless networks. IEEE Trans. Wirel. Commun. 8(4), 1996-2005 (2009)

16. H. Zhang, L. Duan, R. Zhang, in 2019 IEEE International Conference on Communications (ICC). Proactive Eavesdropping of Two Suspicious Communication Links via Jamming, (2019). https://doi.org/10.1109/icc. 2019.8761976

17. J. Xu, L. Duan, R. Zhang, Surveillance and intervention of infrastructure-free mobile communications: a new wireless security paradigm. IEEE Wirel. Commun. 24(4), 152-159 (2017)

18. H. Zhang, L. Duan, in 2018 IEEE Global Communications Conference (GLOBECOM). Going beyond secrecy rate via information jamming, (2018). https://doi.org/10.1109/glocom.2018.8647745

19. H. Lu, H. Zhang, H. Dai, W. Wu, B. Wang, Proactive eavesdropping in UAV-aided suspicious communication systems. IEEE Trans. Veh. Technol. 68(2), 1993-1997 (2019)

20. B. Li, Y. Yao, H. Zhang, Y. Lv, Energy efficiency of proactive cooperative eavesdropping over multiple suspicious communication links. IEEE Trans. Veh. Technol. 68(1), 420-430 (2019)

21. Y. Zeng, X. Xu, R. Zhang, Trajectory design for completion time minimization in UAV-enabled multicasting. IEEE Trans. Wirel. Commun. 17(4), 2233-2246 (2018)

22. C. Li, H. J. Yang, F. Sun, J. M. Cioffi, L. Yang, Multiuser overhearing for cooperative two-way multiantenna relays. IEEE Trans. Veh. Technol. 65(5), 3796-3802 (2016)

23. Z. Bu, H.-J. Li, C. Zhang, J. Cao, A. Li, Y. Shi, Graph K-means based on leader identification, dynamic game, and opinion dynamics. IEEE Trans. Knowl. Data Eng. (Early Access). https://doi.org/10.1109/TKDE.2019.2903712 
24. J. Cao, C. Ding, B. Shi, Motif-based functional backbone extraction of complex networks. Phys. A Stat. Mech. Appl. 526, 121123 (2019)

25. J. Xu, L. Duan, R. Zhang, Proactive eavesdropping via jamming for rate maximization over Rayleigh fading channels. IEEE Wirel. Commun. Lett. 5(1), 80-83 (2016)

26. J. Xu, L. Duan, R. Zhang, Proactive eavesdropping via cognitive jamming in fading channels. IEEE Trans. Wirel. Commun. 16(5), 2790-2806 (2017)

27. C. Zhong, X. Jiang, F. Qu, Z. Zhang, Multi-antenna wireless legitimate surveillance systems: design and performance analysis. Wcsp2017. 16(7), 4585-4599 (2017)

28. H. Cai, Q. Zhang, Z. Li, J. Qin, Proactive monitoring via jamming for rate maximization over MIMO Rayleigh fading channels. IEEE Wirel. Commun. Lett. 21(9), 2021-2024 (2017)

29. R. Zhang, Y.-C. Liang, Exploiting multi-antennas for opportunistic spectrum sharing in cognitive radio networks. IEEE J. Sel. Top. Sig. Process. 2(1), 88-102 (2008)

30. Y. Zeng, R. Zhang, Wireless information surveillance via proactive eavesdropping with spoofing relay. IEEE J. Sel. Top. Sig. Process. 10(8), 1449-1461 (2016)

31. Y. Pei, Y. Liang, L. Zhang, K. Teh, K. Li, Secure communication over MISO cognitive radio channels. IEEE Trans. Wirel. Commun. 9(4), 1494-1502 (2010)

32. L. Zhang, Y. Liang, Y. Xin, Poor V.H., Robust cognitive beamforming with partial channel state information. IEEE Trans. Wirel. Commun. 8(8), 4143-4153 (2009)

33. P. Li, H. Zhang, W. Wu, H. Dai, B. Wang, in 2018 IEEE Global Communications Conference (GLOBECOM). Proactive eavesdropping via jamming in cognitive radio networks, (2018). https://doi.org/10.1109/glocom.2018.8647517

\section{Publisher's Note}

Springer Nature remains neutral with regard to jurisdictional claims in published maps and institutional affiliations.

\section{Submit your manuscript to a SpringerOpen ${ }^{\circ}$ journal and benefit from:}

- Convenient online submission

- Rigorous peer review

- Open access: articles freely available online

- High visibility within the field

- Retaining the copyright to your article

Submit your next manuscript at $\gg$ springeropen.com 